\title{
Mild solutions of fractional evolution equations on an unbounded interval
}

\author{
Zufeng Zhang*, Qun Ning and Hongyan Wang
}

"Correspondence: jshyzzf@163.com School of Mathematics and Statistics, Suzhou University, Suzhou, Anhui 234000, P.R. China

\begin{abstract}
This paper is concerned with the existence results for mild solutions of semilinear fractional evolution equations on an unbounded interval. The methods used in the paper are based on the concept of measure of noncompactness in Fréchet space and classical Tichonov fixed-point theorem. An example is also given to illustrate our main results.
\end{abstract}

MSC: $26 \mathrm{~A} 33 ; 26 \mathrm{~A} 42$

Keywords: unbounded interval; fractional evolution equations; mild solution; measure of noncompactness

\section{Introduction}

We consider the following form for the fractional evolution equations:

$$
\left\{\begin{array}{l}
D^{q} x(t)=A x(t)+f(t, x(t)), \quad t \in \mathbf{R}^{+}:=[0, \infty), \\
x(0)=x_{0}
\end{array}\right.
$$

where $D^{q}$ is the Caputo fractional derivative of order $0<q<1, A$ is the infinitesimal generator of a strongly continuous semigroup of bounded linear operator $\{T(t)\}_{t \geq 0}$ in Banach space $E$, and $f: \mathbf{R}^{+} \times E \rightarrow E$ is a given function.

Fractional differential equations have appeared in many branches of physics, economics, and technical sciences $[1,2]$. There has been a considerable developments in fractional differential equations in the last decades. Recently, the definition for mild solutions of fractional evolution equations was successfully given in two ways: one was given by using some probability densities [3-6], the other was given by the so-called solution operator [7-10]. In the scheme of these definitions, many interesting existence results for mild solutions were established by various fixed-point theorems.

We notice that all the papers mentioned above were investigated mild solutions on a bounded interval. On the other hand, research on mild solutions on an unbounded interval of the integer order evolution equations could be found in papers [11,12] and the references therein. Very recently, Banaś, O'Regan [13] studied existence and attractiveness of solutions of a nonlinear quadratic integral equations of fractional order on an unbounded interval. By means of Darbo's fixed-point theorem, Su [14] considered the existence of solutions to boundary value problems of fractional differential equations on unbounded domains. So we think there is a real need to concern existence results for fractional evolution equation on an unbounded interval. But as far as we known, there are few works on

C2014 Zhang et al.; licensee Springer. This is an Open Access article distributed under the terms of the Creative Commons Attribution License (http://creativecommons.org/licenses/by/2.0), which permits unrestricted use, distribution, and reproduction in any medium, provided the original work is properly cited. 
this subject up to now. Motivated by this, we concern ourselves with existence results for mild solutions of problem (1.1) in the present paper by the Tichonov fixed-point theorem.

The rest of paper will be organized as follows. In Section 2 we will introduce some basic definitions and lemmas from the measure of noncompactness, fractional derivation, and integration. Section 3 is devoted to the existence results for problem (1.1). We shall present in Section 4 an example which illustrates our main theorems.

\section{Preliminaries}

In this section, we collect some definitions and results which will be used in the rest of the paper. Let $(E,\|\cdot\|)$ be a real Banach space. Define $L^{p}([0, b], E)$ be the space of $E$-valued Bochner functions on $[0, b]$ with the norm $\|x\|_{L^{p}[0, b]}=\left(\int_{0}^{b}\|x(s)\|^{p} d s\right)^{\frac{1}{p}}, 1 \leq p<\infty$. Denote by $C\left(\mathbf{R}^{+}, E\right)$ the space of continuous functions from $\mathbf{R}^{+}$into $E$.

Definition 2.1 ([2]) The Riemann-Liouville fractional integral of order $q \in \mathbf{R}^{+}$of a function $f: \mathbf{R}^{+} \rightarrow E$ is defined by

$$
I_{0}^{q} f(t)=\frac{1}{\Gamma(q)} \int_{0}^{t}(t-s)^{q-1} f(s) d s, \quad t>0,
$$

provided the right-hand side is pointwise defined on $\mathbf{R}^{+}$, where $\Gamma$ is the gamma function.

Definition 2.2 ([2]) The Caputo fractional derivative of order $0<q<1$ of a function $f \in$ $C^{1}\left(\mathbf{R}^{+} ; E\right)$ is defined by

$$
D^{q} f(t)=\frac{1}{\Gamma(1-q)} \int_{0}^{t}(t-s)^{-q} f^{\prime}(s) d s, \quad t>0
$$

The space $C\left(\mathbf{R}^{+}, E\right)$ is the locally convex Fréchet space of continuous functions with the metric

$$
d(x, y)=\sup \left\{2^{n} \frac{\|x-y\|_{n}}{1+\|x-y\|_{n}}: n=1,2, \ldots\right\}
$$

where $\|x\|_{n}=\sup \{\|x(t)\|: t \in[0, n]\}$.

According to [11], a sequence $\left\{x_{n}\right\}$ is convergent to $x$ in $C\left(\mathbf{R}^{+}, E\right)$ if and only if $\left\{x_{n}\right\}$ is uniformly convergent to $x$ on compact subsets of $\mathbf{R}^{+}$. Moreover, a subset $X \subset C\left(\mathbf{R}^{+}, E\right)$ is relatively compact if and only if the restrictions on $[0, T]$ of all functions from $X$ form an equicontinous set for each $T>0$ and $X(t)$ is relatively compact in $E$ for each $t \in \mathbf{R}^{+}$, where $X(t)=\{x(t): x \in X\}$.

Next, we present some basic facts concerning the measure of noncompactness on $C\left(\mathbf{R}^{+}, E\right)$. Let $\mathbf{0}$ be the zero element of $E$. Denote by $B(x, r)$ the closed ball centered at $x$ with radius and by $B_{r}$ the ball $B(\mathbf{0}, r)$. If $X$ is a subset of $E$, then the symbols $\bar{X}$ and $\operatorname{Conv} X$ stand for the closure and convex closure of $X$, respectively. Further we assume $\mathfrak{M}_{E}$ to be the family of all nonempty and bounded subsets of $E, \mathfrak{N}_{E}$ represents its subfamily consisting of relatively compact sets.

Definition 2.3 ([15]) A function $\mu: \mathfrak{M}_{E} \rightarrow \mathbf{R}^{+}$is said to be of regular noncompactness if it satisfies the following conditions: 
(i) $\mu(X)=0 \Leftrightarrow X \in \mathfrak{N}_{E}$.

(ii) $X \subset Y \Rightarrow \mu(X) \leq \mu(Y)$.

(iii) $\mu(\operatorname{Conv} X)=\mu(X)$.

(iv) $\mu(\lambda X+(1-\lambda) Y) \leq \lambda \mu(X)+(1-\lambda) \mu(Y)$ for $\lambda \in[0,1]$.

(v) $\mu(\lambda X)=|\lambda| \mu(X)$ for $\lambda \in \mathbf{R}$

(vi) $\mu(X+Y) \leq \mu(X)+\mu(Y)$

(vii) $\mu(X \cup Y)=\max \{\mu(X), \mu(Y)\}$.

(viii) If $\left\{X_{n}\right\}$ is a sequence of nonempty, bounded, and closed subsets of $E$ such that $X_{n+1} \subset X_{n}(n=1,2, \ldots)$ and if $\lim _{n \rightarrow \infty} \mu\left(X_{n}\right)=0$, then the intersection $X_{\infty}=\bigcap_{n=1}^{\infty} X_{n}$ is nonempty.

Next we consider the measure of noncompactness in $C\left(\mathbf{R}^{+}, E\right)$ introduced in [16]. To this end, let $r: \mathbf{R}^{+} \rightarrow(0, \infty)$ be a given function and

$$
\mathfrak{M}_{r}=\left\{X \subset C\left(\mathbf{R}^{+}, E\right): X \neq \emptyset,\|x(t)\| \leq r(t) \text { for } x \in X, t \geq 0\right\}
$$

Denote by $\mathfrak{N}_{r}$ the family of all relatively compact members of $\mathfrak{M}_{r}$.

Fix $X \in \mathfrak{M}_{r}$ and $T>0$; for $x \in X$ and $\epsilon>0$, denote by $\omega^{T}(x, \epsilon)$ the modulus of continuity of the function $x$ on the interval $[0, T]$ as follows:

$$
\omega^{T}(x, \epsilon)=\sup \{\|x(t)-x(s)\|: t, s \in[0, T],|t-s| \leq \epsilon\} .
$$

Further, we define

$$
\omega^{T}(X, \epsilon)=\sup \left\{\omega^{T}(x, \epsilon), x \in X\right\}, \quad \omega_{0}^{T}(X)=\lim _{\epsilon \rightarrow 0^{+}} \omega^{T}(X, \epsilon) .
$$

Remark 2.4 ([15]) We observe that functions from the set $X \in \mathfrak{M}_{r}$ are equicontinuous on compact intervals of $\mathbf{R}^{+}$if and only if $\omega_{0}^{T}(X)=0$ for each $T>0$.

Assume that $\mu$ is the regular measure of noncompactness in $E$ and let us put $\bar{\mu}^{T}(X)=$ $\sup \{\mu(X(t)): t \in[0, T]\}$; we define the $\gamma$ on the family $\mathfrak{M}_{r}$ by

$$
\gamma_{R}(X)=\sup \left\{\frac{1}{R(T)}\left(\omega_{0}^{T}(X), \bar{\mu}^{T}(X)\right): T \geq 0\right\},
$$

where $R: \mathbf{R}^{+} \rightarrow(0, \infty)$ is a given function such that $r(t) \leq R(t)$ for $t \geq 0$.

Theorem 2.5 ([11]) The mapping $\gamma_{R}: \mathfrak{M}_{r} \rightarrow \mathbf{R}^{+}$has the following properties:

(1) The family $\operatorname{ker} \gamma_{R}=\left\{X \in \mathfrak{M}_{r}: \gamma_{R}(X)=0\right\}=\mathfrak{N}_{r}$.

(2) $\gamma_{R}(\operatorname{Conv}(X))=\gamma_{R}(X)$.

(3) If $\left\{X_{n}\right\}$ is a sequence of closed sets from $\mathfrak{M}_{r}$ such that $X_{n+1} \subset X_{n}(n=0,1, \ldots)$ and if $\lim _{n \rightarrow \infty} \gamma_{R}\left(X_{n}\right)=0$, then the intersection $X_{\infty}=\bigcap_{n=1}^{\infty} X_{n}$ is nonempty.

For $X \in \mathfrak{M}_{r}$, let us denote $\int_{0}^{t} X(\tau) d \tau=\left\{\int_{0}^{t} x(\tau) d \tau, x \in X\right\}$.

Lemma 2.6 ([17]) If all functions belonging to $X$ are equicontinuous on compact subsets of $\mathbf{R}^{+}$then

$$
\mu\left(\int_{0}^{t} X(\tau)\right) \leq \int_{0}^{t} \mu(X(\tau)) d \tau \quad \text { for } t \geq 0 .
$$


Lemma 2.7 ([15]) If $\mu$ is a regular measure of noncompactness then

$$
|\mu(X)-\mu(Y)| \leq \mu(B(\mathbf{0}, 1)) d_{H}(X, Y)
$$

for any bounded subset $X, Y \subset E$, where $d_{H}$ is the Hausdorff distance between $X$ and $Y$.

Lemma 2.8 ([18]) Suppose that $x \geq 1$, then

$$
\left(\frac{x}{e}\right)^{x} \sqrt{2 \pi x}\left(1+\frac{1}{12 x}\right)<\Gamma(x+1)<\left(\frac{x}{e}\right)^{x} \sqrt{2 \pi x}\left(1+\frac{1}{12 x-0.5}\right) .
$$

Similar to Cauchy's formula, we have the following lemma.

Lemma 2.9 If $z: \mathbf{R}^{+} \rightarrow \mathbf{R}$ is a continuous function and $q>0$, then

$$
\begin{aligned}
& \int_{0}^{t}\left(t-s_{1}\right)^{q-1} \int_{0}^{s_{1}}\left(s_{1}-s_{2}\right)^{q-1} \cdots \int_{0}^{s_{n}}\left(s_{n}-s_{n+1}\right)^{q-1} z\left(s_{n+1}\right) d s_{n+1} d s_{n} \cdots d s_{1} \\
& \quad=\frac{\Gamma^{n+1}(q)}{\Gamma((n+1) q)} \int_{0}^{t}(t-s)^{(n+1) q-1} z(s) d s .
\end{aligned}
$$

Proof By changing the integral order and some calculations, one can prove the lemma easily. We omit the proof here.

Theorem 2.10 ([19], Tikhonov fixed-point theorem) Let $V$ be a locally convex topological vector space. For any nonempty compact convex $X$ in $V$, if the function $F: X \rightarrow X$ is continuous, then $F$ has a fixed point in $X$.

\section{Main results}

In this section we will establish the existence results. Based on reference [4], we give the definition of the mild solutions of problem (1.1) as follows.

Definition 3.1 A continuous function $x: \mathbf{R}^{+} \rightarrow E$ is said to be a mild solution of (1.1) if $x$ satisfies

$$
x(t)=\mathfrak{S}(t) x_{0}+\int_{0}^{t}(t-s)^{q-1} \mathfrak{T}(t-s) f(s, x(s)) d s,
$$

where

$$
\begin{aligned}
\mathfrak{S}(t) & =\int_{0}^{\infty} \xi_{q}(\theta) T\left(t^{q} \theta\right) d \theta \\
\mathfrak{T}(t) & =q \int_{0}^{\infty} \theta \xi_{q}(\theta) T\left(t^{q} \theta\right) d \theta \\
\xi_{q}(\theta) & =\frac{1}{q} \theta^{-1-\frac{1}{q}} \Psi_{q}\left(\theta^{-\frac{1}{q}}\right), \\
\Psi_{q}(\theta) & =\frac{1}{\pi} \sum_{n=1}^{\infty}(-1)^{n-1} \theta^{-q n-1} \frac{\Gamma(n q+1)}{n !} \sin (n \pi q), \quad \theta \in \mathbf{R}^{+} .
\end{aligned}
$$


Remark $3.2([20]) \xi_{q}(\theta)$ is the probability density function defined on $\mathbf{R}^{+}$and

$$
\int_{0}^{\infty} \theta \xi_{q}(\theta) d \theta=\int_{0}^{\infty} \frac{1}{\theta^{q}} \Psi_{q}(\theta) d \theta=\frac{1}{\Gamma(1+q)} .
$$

To state and prove our main results for the existence of mild solutions of problem (1.1), we need the following hypotheses:

(H1) The $C_{0}$-semigroup $\{T(t)\}_{t>0}$ generated by $A$ is compact and there exists a constant $M>0$ such that $M=\sup \left\{\|T(t)\| ; t \in \mathbf{R}^{+}\right\}<+\infty$.

(H2) The function $f: \mathbf{R}^{+} \times E \rightarrow E$ satisfies the Carathéodory type conditions, i.e. $f(t, \cdot): E \rightarrow E$ is continuous for a.e. $t \in \mathbf{R}^{+}$and $f(\cdot, x): \mathbf{R}^{+} \rightarrow E$ is strongly measurable for each $x \in E$.

(H3) There exists a locally $L^{\frac{1}{p}}$-integrable $(0<p<q)$ function $m: \mathbf{R}^{+} \rightarrow \mathbf{R}^{+}$such that $\|f(t, x)\| \leq m(t)$ for all $x \in E$ and a.e. $t \in \mathbf{R}^{+}$.

(H4) $k: \mathbf{R}^{+} \rightarrow \mathbf{R}^{+}$is a measurable and essentially bounded function on the compact intervals of $\mathbf{R}^{+}$such that

$$
\mu(f(t, X)) \leq k(t) \mu(X)
$$

for a.e. $t \in \mathbf{R}^{+}$and bounded subsets $X$ of $E$, where $\mu$ is a regular measure of noncompactness on $E$.

Remark 3.3 If $\|f(t, x)-f(t, y)\| \leq L(t)\|x-y\|, L(t) \in L^{1}\left(\mathbf{R}^{+}, \mathbf{R}^{+}\right), x, y \in E$, then we get $\alpha(f(t, X)) \leq L(t) \alpha(X)$ for each bounded $X \subset E$ and a.e. $t \in \mathbf{R}^{+}$.

Lemma 3.4 Assume that hypotheses (H1)-(H3) hold, then:

(i) For any fixed $t \geq 0, \mathfrak{S}(t)$ and $\mathfrak{T}(t)$ defined in (3.1) are linear and bounded operators, i.e. for any $x \in E$,

$$
\|\mathfrak{S}(t) x\| \leq M\|x\|, \quad\|\mathfrak{T}(t) x\| \leq \frac{M}{\Gamma(q)}\|x\| .
$$

(ii) $\mathfrak{S}(t)$ and $\mathfrak{T}(t)$ are continuous in the uniform operator topology for $t>0$.

Proof (i) was proved in [6] and (ii) can easily be proved by the compactness of the semigroup $\{T(t)\}_{t>0}$. We omit the proof here.

Theorem 3.5 Under the assumptions (H1)-(H4) problem (1.1) has at least one mild solution $x$ in $C\left(\mathbf{R}^{+}, E\right)$ for each $x_{0} \in E$.

Proof Define operator $F: C\left(\mathbf{R}^{+}, E\right) \rightarrow C\left(\mathbf{R}^{+}, E\right)$ by

$$
(F x)(t)=\mathfrak{S}(t) x_{0}+\int_{0}^{t}(t-s)^{q-1} \mathfrak{T}(t-s) f(s, x(s)) d s, \quad t \geq 0 .
$$

Firstly, we shall show that there exists a function $r: \mathbf{R}^{+} \rightarrow(0, \infty)$ such that if $x \in C\left(\mathbf{R}^{+}, E\right)$ and $\|x(t)\| \leq r(t)$ for $t \geq 0$, then

$$
\|(F x)(t)\| \leq r(t)
$$


In fact, we choose $r(t)=M\left\|x_{0}\right\|+\frac{M}{\Gamma(q)}\left(\frac{1-p}{q-p}\right)^{1-p} t^{q-p}\|m\|_{L^{\frac{1}{p}}[0, t]}$, then from the hypotheses we have

$$
\begin{aligned}
\|(F x)(t)\| \leq & \left\|\mathfrak{S}(t) x_{0}\right\|+\left\|\int_{0}^{t}(t-s)^{q-1} \mathfrak{T}(t-s) f(s, x(s)) d s\right\| \\
= & \left\|\int_{0}^{\infty} \xi_{q}(\theta) T\left(t^{q} \theta\right) x_{0} d \theta\right\| \\
& +\left\|q \int_{0}^{t}(t-s)^{q-1} \int_{0}^{\infty} \theta \xi_{q}(\theta) T\left((t-s)^{q} \theta\right) d \theta f(s, x(s)) d s\right\| \\
\leq & M\left\|x_{0}\right\|+\frac{M}{\Gamma(q)} \int_{0}^{t}(t-s)^{q-1} m(s) d s \\
\leq & M\left\|x_{0}\right\|+\frac{M}{\Gamma(q)}\left(\int_{0}^{t}(t-s)^{\frac{q-1}{1-p}} d s\right)^{1-p}\|m\|_{L^{\frac{1}{p}}[0, t]} \\
\leq & M\left\|x_{0}\right\|+\frac{M}{\Gamma(q)}\left(\frac{1-p}{q-p}\right)^{1-p} t^{q-p}\|m\|_{L^{\frac{1}{p}}[0, t]} \\
& =r(t) .
\end{aligned}
$$

Moreover, $r(t)$ is nondecreasing.

Let us fix $x \in C\left(\mathbf{R}^{+}, E\right)$ such that $\|x(t)\| \leq r(t)$, we will estimate the modulus of continuity of the function $F x$. Fix arbitrary $T \geq 0$ and $\epsilon \geq 0$ and take $t_{1}, t_{2} \in[0, T]$ such that $\left|t_{2}-t_{1}\right| \leq \epsilon$. Without loss of generality, we assume that $t_{2} \geq t_{1}$, then

$$
\begin{aligned}
& \left\|(F x)\left(t_{2}\right)-(F x)\left(t_{1}\right)\right\| \\
& =\| \mathfrak{S}\left(t_{2}\right) x_{0}+\int_{0}^{t_{2}}\left(t_{2}-s\right)^{q-1} \mathfrak{T}\left(t_{2}-s\right) f(s, x(s)) d s \\
& -\mathfrak{S}\left(t_{1}\right) x_{0}+\int_{0}^{t_{1}}\left(t_{1}-s\right)^{q-1} \mathfrak{T}\left(t_{1}-s\right) f(s, x(s)) d s \| \\
& \leq\left\|\mathfrak{S}\left(t_{2}\right) x_{0}-\mathfrak{S}\left(t_{1}\right) x_{0}\right\|+\left\|\int_{t_{1}}^{t_{2}}\left(t_{2}-s\right)^{q-1} \mathfrak{T}\left(t_{2}-s\right) f(s, x(s)) d s\right\| \\
& +\left\|\int_{0}^{t_{1}}\left[\left(t_{2}-s\right)^{q-1}-\left(t_{1}-s\right)^{q-1}\right] \mathfrak{T}\left(t_{2}-s\right) f(s, x(s)) d s\right\| \\
& +\left\|\int_{0}^{t_{1}}\left(t_{1}-s\right)^{q-1}\left[\mathfrak{T}\left(t_{2}-s\right)-\mathfrak{T}\left(t_{1}-s\right)\right] f(s, x(s)) d s\right\| \\
& \leq \omega^{T}(\mathfrak{S}, \epsilon)\left\|x_{0}\right\|+\frac{M}{\Gamma(q)} \int_{t_{1}}^{t_{2}}\left(t_{2}-s\right)^{q-1} m(s) d s \\
& +\frac{M}{\Gamma(q)} \int_{0}^{t_{1}}\left[\left(t_{1}-s\right)^{q-1}-\left(t_{2}-s\right)^{q-1}\right] m(s) d s \\
& +\int_{0}^{t_{1}}\left(t_{1}-s\right)^{q-1}\left\|\mathfrak{T}\left(t_{2}-s\right)-\mathfrak{T}\left(t_{1}-s\right)\right\| m(s) d s \\
& \leq \omega^{T}(\mathfrak{S}, \epsilon)\left\|x_{0}\right\|+\frac{M}{\Gamma(q)}\left(\frac{1-p}{q-p}\right)^{1-p} \epsilon^{q-p}\|m\|_{L^{\frac{1}{p}}\left[t_{1}, t_{2}\right]} \\
& +\frac{M}{\Gamma(q)}\left(\int_{0}^{t_{1}}\left[\left(t_{1}-s\right)^{q-1}-\left(t_{2}-s\right)^{q-1}\right]^{\frac{1}{1-p}} d s\right)^{1-p}\|m\|_{L^{\frac{1}{p}}\left[0, t_{1}\right]}
\end{aligned}
$$




$$
\begin{aligned}
& +v^{T}(\mathfrak{T}, \epsilon) \int_{0}^{t_{1}}\left(t_{1}-s\right)^{q-1} m(s) d s \\
\leq & \omega^{T}(\mathfrak{S}, \epsilon)\left\|x_{0}\right\|+\frac{M}{\Gamma(q)}\left(\frac{1-p}{q-p}\right)^{1-p} \epsilon^{q-p} \sup \left\{\|m\|_{\left.L^{\frac{1}{p}}{ }_{\left[t_{1}, t_{2}\right]}: 0 \leq t_{1} \leq t_{2} \leq T\right\}}\right. \\
& +\frac{M}{\Gamma(q)}\left(\frac{1-p}{q-p}\right)^{1-p} \epsilon^{q-p}\|m\|_{L^{\frac{1}{p}}[0, T]} \\
& +v^{T}(\mathfrak{T}, \epsilon)\left(\frac{1-p}{q-p}\right)^{1-p} T^{q-p}\|m\|_{L^{\frac{1}{p}}[0, T]} \\
:= & \Omega(T, \epsilon),
\end{aligned}
$$

where $\omega^{T}(\mathfrak{S}, \epsilon)=\sup \left\{\left\|\mathfrak{S}\left(t_{2}\right)-\mathfrak{S}\left(t_{1}\right)\right\|: t_{1}, t_{2} \in[0, T],\left|t_{2}-t_{1}\right| \leq \epsilon\right\}, v^{T}(\mathfrak{T}, \epsilon)=\sup \left\{\| \mathfrak{T}\left(t_{2}\right)-\right.$ $\left.\mathfrak{T}\left(t_{1}\right) \|: t_{1}, t_{2} \in[0, T],\left|t_{2}-t_{1}\right| \leq \epsilon\right\}$.

Therefore, we have

$$
\left\|(F x)\left(t_{2}\right)-(F x)\left(t_{1}\right)\right\| \leq \Omega(T, \epsilon)
$$

for $x$ such that $\|x(t)\| \leq r(t)$. From Lemma 3.4, we have

$$
\lim _{\epsilon \rightarrow 0^{+}} \Omega(T, \epsilon)=0 \quad \text { for } T \geq 0
$$

Now define the subset $Q$ of $C\left(\mathbf{R}^{+}, E\right)$ as follows:

$$
Q=\left\{x \in C\left(\mathbf{R}^{+}, E\right):\|x(t)\| \leq r(t), \omega^{T}(x, \epsilon) \leq \Omega(T, \epsilon) \text { for } t, T, \epsilon \geq 0\right\} .
$$

In view of $x(t) \equiv M x_{0} \in Q$, we see that $Q$ is nonempty. Moreover, $Q$ is a closed and convex subset of $C\left(\mathbf{R}^{+}, E\right)$. From (3.4) and Remark 2.4, we find that the set $Q$ is the family consisting of functions equicontinuous on compact intervals of $\mathbf{R}^{+}$. By (3.2) we find that $F$ maps $Q$ into itself.

Next, we will show that $F: Q \rightarrow Q$ is continuous. For $x, x_{n} \in Q$ such that $\lim _{n \rightarrow \infty} x_{n}=x$ in $C\left(\mathbf{R}^{+}, E\right)$, we have

$$
\lim _{n \rightarrow \infty} \sup _{t \leq T}\left\|x_{n}(t)-x(t)\right\|=0, \quad T \geq 0 .
$$

Fix $T \geq 0$; then we get

$$
\begin{aligned}
\sup _{t \leq T}\left\|\left(F x_{n}\right)(t)-(F x)(t)\right\| & \leq \sup _{t \leq T} \int_{0}^{t}(t-s)^{q-1}\left\|\mathfrak{T}(t-s)\left[f\left(s, x_{n}(s)\right)-f(s, x(s))\right]\right\| d s \\
& \leq \frac{M}{\Gamma(q)} \sup _{t \leq T} \int_{0}^{t}(t-s)^{q-1}\left\|f\left(s, x_{n}(s)\right)-f(s, x(s))\right\| d s .
\end{aligned}
$$

Hence $\lim _{n \rightarrow \infty} F x_{n}=F x$ in $C\left(\mathbf{R}^{+}, E\right)$ by the Lebesgue dominated convergence theorem and hypothesis (H2).

Let $Q_{0}=Q, Q_{n}=\operatorname{Conv} F\left(Q_{n-1}\right)$ for $n=1,2, \ldots$, then all sets of this sequence are nonempty, closed, and convex. Moreover, $Q_{n+1} \subset Q_{n}$ for $n=0,1, \ldots$ By the equicontinuity of the set $Q$ on compact intervals, we have

$$
\omega_{0}^{T}\left(Q_{n}\right)=0, \quad \text { for } n=0,1, \ldots, \text { and } T \geq 0 .
$$


Set $z_{n}(t)=\mu\left(Q_{n}(t)\right)$. From Lemma 2.7 and (3.3) we have

$$
\left|z_{n}(t)-z_{n}(s)\right| \leq \mu(B(\mathbf{0}, 1)) \Omega(T,|t-s|),
$$

which together with (3.4) implies the continuity of $z_{n}(t)$ on $\mathbf{R}^{+}$.

By the properties of $\mu$, Lemma 2.6, and Hypothesis (H4) we have

$$
\begin{aligned}
z_{n+1}(t) & =\mu\left(\left(\operatorname{Conv} F Q_{n}\right)(t)\right) \\
& =\mu\left(\int_{0}^{t}(t-s)^{q-1} \mathfrak{T}(t-s) f\left(s, Q_{n}(s)\right) d s\right) \\
& \leq M \int_{0}^{t}(t-s)^{q-1} k(s) z_{n}(s) d s \\
& \leq M \bar{k}(t) \int_{0}^{t}(t-s)^{q-1} z_{n}(s) d s,
\end{aligned}
$$

where $\bar{k}(t)=\operatorname{ess} \sup \{k(s): s \leq t\}$, obviously, $\bar{k}(t)$ is nondecreasing.

By the method of mathematical induction and Lemma 2.9, we have

$$
\begin{aligned}
z_{n+1}(t) \leq & M \bar{k}(t) \int_{0}^{t}(t-s)^{q-1} z_{n}(s) d s \\
\leq & M^{2} \bar{k}^{2}(t) \int_{0}^{t}\left(t-s_{1}\right)^{q-1} \int_{0}^{s_{1}}\left(s_{1}-s_{2}\right)^{q-1} z_{n-1}\left(s_{2}\right) d s_{2} d s_{1} \\
\leq & M^{n+1} \bar{k}^{n+1}(t) \int_{0}^{t}\left(t-s_{1}\right)^{q-1} \int_{0}^{s_{1}}\left(s_{1}-s_{2}\right)^{q-1} \cdots \\
& \times \int_{0}^{s_{n}}\left(s_{n}-s_{n+1}\right)^{q-1} z_{0}\left(s_{n+1}\right) d s_{n+1} d s_{n} \cdots d s_{1} \\
\leq & M^{n+1} \bar{k}^{n+1}(t) \frac{\Gamma^{n+1}(q)}{\Gamma((n+1) q)} \int_{0}^{t}(t-s)^{(n+1) q-1} z_{0}(s) d s .
\end{aligned}
$$

Then for $n \geq \frac{1}{q}$ we have

$$
z_{n+1} \leq M^{n+1} \bar{k}^{n+1}(t) \frac{\Gamma^{n+1}(q)}{\Gamma((n+1) q)} t^{(n+1) q-1} \int_{0}^{t} z_{0}(s) d s
$$

Now we use the measure of noncompactness $\gamma_{R}$ defined in $C\left(\mathbf{R}^{+}, E\right)$ by formula $(2.1)$, where

$$
R(t)=r(t)\left(1+(M \Gamma(q) \bar{k}(t))^{\frac{1}{q}}\right)\left(1+\int_{0}^{t} z_{0}(s) d s\right) e^{(M \Gamma(q) \bar{k}(t))^{\frac{1}{q}} t}
$$

Obviously $r(t) \leq R(t)$. By (3.5), for $n \geq \frac{1}{q}$ we have

$$
\bar{\mu}^{T}\left(Q_{n+1}\right)=\sup _{t \leq T} z_{n+1} \leq M^{n+1} \bar{k}^{n+1}(T) \frac{\Gamma^{n+1}(q)}{\Gamma((n+1) q)} T^{(n+1) q-1} \int_{0}^{T} z_{0}(s) d s
$$


and

$$
\begin{aligned}
\frac{\bar{\mu}^{T}\left(Q_{n+1}\right)}{R(T)}= & \frac{(M \Gamma(q) \bar{k}(T))^{n+1-\frac{1}{q}} T^{(n+1) q-1}}{r(T) \Gamma((n+1) q) e^{(M \Gamma(q) \bar{k}(T))^{\frac{1}{q}} T}} \\
= & \frac{\left((M \Gamma(q) \bar{k}(T))^{\frac{1}{q}} T\right)^{(n+1) q-1}}{r(T) \Gamma((n+1) q) e^{(M \Gamma(q) \bar{k}(T))^{\frac{1}{q}} T}} .
\end{aligned}
$$

By the estimation $\sup \left\{\frac{a^{n}}{e^{a}}: a \geq 0\right\} \leq \frac{n^{n}}{e^{n}}$ we have

$$
\frac{\bar{\mu}^{T}\left(Q_{n+1}\right)}{R(T)} \leq \frac{((n+1) q-1)^{(n+1) q-1}}{r(T) \Gamma((n+1) q) e^{(n+1) q-1}} .
$$

Then from Lemma 2.8, we get, for $n>\frac{2}{q}$,

$$
\frac{\bar{\mu}^{T}\left(Q_{n+1}\right)}{R(T)} \leq \frac{1}{r(T) \sqrt{2 \pi((n+1) q-1)}}
$$

Hence we have

$$
\lim _{n \rightarrow \infty} \gamma_{R}\left(Q_{n+1}\right)=\lim _{n \rightarrow \infty} \sup \left\{\frac{1}{R(T)}\left(\omega_{0}^{T}\left(Q_{n+1}\right), \bar{\mu}^{T}\left(Q_{n+1}\right)\right): T \geq 0\right\}=0 .
$$

In view of Theorem 2.5 we get $Q_{\infty}=\bigcap_{n=0}^{\infty} Q_{n} \neq \emptyset$. Since $0 \leq \gamma\left(Q_{\infty}\right) \leq \lim _{n \rightarrow \infty} \gamma_{R}\left(Q_{n}\right)$, we have $\gamma\left(Q_{\infty}\right)=0$, which implies $Q_{\infty}$ is a compact subset in $C\left(\mathbf{R}^{+}, E\right)$.

Consider $F: Q_{\infty} \rightarrow Q_{\infty}$. From the above arguments, we see that all the conditions of the Tichonov fixed-point theorem are satisfied. Therefore $F$ has at least one fixed point $x$ in $Q_{\infty}$, which is the mild solution of problem (1.1). The proof is completed.

\section{An example}

In this section, we give an example to illustrate the applications of Theorem 3.5 established in the previous sections.

Let $\Omega \in \mathbf{R}^{n}$ be a bounded domain with smooth boundary $\partial \Omega$. Consider a fractional initial/boundary value Cauchy problem of the form

$$
\left\{\begin{array}{l}
D^{q} u(t, z)=u_{z z}(t, z)+f(t, u(t, z)), \quad t \in \mathbf{R}^{+}, z \in \Omega, \\
u(0, z)=u_{0}, \quad z \in \Omega, \\
u(t, z)=0, \quad t \in \mathbf{R}^{+}, z \in \partial \Omega,
\end{array}\right.
$$

where $D^{q}$ is the Caputo fractional partial derivative of order $0<q<1$, and $f$ is a given function.

Let $E=L^{2}(\Omega)$, we define an operator $A u=\frac{\partial^{2} u}{\partial z^{2}}$ on $E$ with the domain

$$
D(A)=\left\{u \in E: u, \frac{\partial u}{\partial z} \text { are absolutely continuous, } \frac{\partial^{2} u}{\partial z^{2}} \in E, u=0 \text { on } \partial \Omega\right\} .
$$

It is well known that $A$ generates a strongly continuous semigroup $\{T(t)\}_{t \geq 0}$ which is compact, analytic, and self-adjoint. 
Then the system (4.1) can be reformulated as follows in $E$ :

$$
\left\{\begin{array}{l}
D^{q} x(t)=A x(t)+f(t, x(t)), \quad t \in \mathbf{R}^{+}, \\
x(0)=u_{0}
\end{array}\right.
$$

where $x(t)=u(t, \cdot)$, that is, $x(t) z=u(t, z), z \in \Omega$.

Let us take $q=\frac{1}{2}, f(t, x(t))=\frac{1}{t^{\frac{1}{4}}} \sin x(t)$. Firstly, we see that (H1)-(H3) are satisfied. From $\|f(t, x(t))-f(t, y(t))\| \leq \frac{1}{t^{\frac{1}{4}}}\|x-y\|_{\infty}$ and Remark 3.3 we find that $(\mathrm{H} 4)$ is satisfied. According to Theorem 3.5, problem (4.1) has at least one mild solution in $C\left(\mathbf{R}^{+}, E\right)$.

\section{Competing interests}

The authors declare that they have no competing interests.

\section{Authors' contributions}

All authors contributed equally to the manuscript. All authors read and approved the final version.

\section{Acknowledgements}

This work was partially supported by the Professor (Doctor) Scientific Research Foundation of Suzhou University (2013jb04), the Nature Science Foundation of Anhui Provincial Education (KJ2013A248, KJ2012Z403).

Received: 19 August 2013 Accepted: 16 December 2013 Published: 22 Jan 2014

\section{References}

1. Oldham, KB, Spanier, J: The Fractional Calculus. Academic Press, New York (1974)

2. Podlubny, I: Fractional Differential Equation. Academic Press, San Diego (1999)

3. El-Borai, MM: Some probability densities and fundamental solutions of fractional evolution equations. Chaos Solitons Fractals 14, $433-440$ (2002)

4. Zhou, Y, Jiao, F: Nonlocal Cauchy problem for fractional evolution equations. Nonlinear Anal. 11, 4465-4475 (2010)

5. Cao, J, Yang, Q, Huang, Z: Optimal mild solutions and weighted pseudo-almost periodic classical solutions of fractional integro-differential equations. Nonlinear Anal. 74, 224-234 (2011)

6. Wang, J, Zhou, Y: A class of fractional evolution equations and optimal controls. Nonlinear Anal. 12, 262-272 (2011)

7. Shu, $X$, Lai, $Y, C$ Chen, $Y$ : The existence of mild solutions for impulsive fractional partial differential equations. Nonlinear Anal. 74, 2003-2011 (2011)

8. Cuevas, C, Lizama, C: Almost automorphic solutions to a class of semilinear fractional differential equations. Appl. Math. Lett. 21, 1315-1319 (2008)

9. Agarwal, R, Andrade, B, Cuevas, C: Weighted pseudo-almost periodic solutions of a class of semilinear fractional differential equations. Nonlinear Anal. 11, 3532-3554 (2011)

10. Lizama, C: An operator theoretical approach to a class of fractional order differential equations. Appl. Math. Lett. 24 184-190 (2011)

11. Olszowy, L, Wedrychowicz, S: Mild solutions of semilinear evolution equation on an unbounded interval and their applications. Nonlinear Anal. 72, 2119-2126 (2010)

12. Jawahdou, A: Mild solutions of functional semilinear evolution Volterra integrodifferential equations on an unbounded interval. Nonlinear Anal. 74, 7325-7332 (2011)

13. Banaś, J, O'Regan, D: On existence and local attractivity of solutions of a quadratic Volterra integral equation of fractional order. J. Math. Anal. Appl. 345, 573-582 (2008)

14. Su, X: Solutions to boundary value problem of fractional order on unbounded domains in a Banach space. Nonlinear Anal. 74, 2844-2852 (2011)

15. Banaś, J, Goebel, K: Measures of Noncompactness in Banach Spaces. Lecture Notes in Pure and Applied Math., vol. 60. Dekker, New York (1980)

16. Olszowy, L: On existence of solutions of a quadratic Urysohn integral equation on an unbounded interval. Comment. Math. 48(1), 103-112 (2008)

17. Olszowy, L: Solvability of some functional integral equation. Dyn. Syst. Appl. 18, 667-676 (2009)

18. Wang, F, Zhao, Y: A two-sided inequality of gamma function. J. Math. Res. Expo. 27, 667-670 (2007)

19. Corduneanu, C: Integral Equations and Applications. Cambridge University Press, New York (1990)

20. Mainardi, F, Paradisi, P, Gorenflo, R: Probability distributions generated by fractional diffusion equations. In: Kertesz, J, Kondor, I (eds.) Econophysics: An Emerging Science. Kluwer, Dordrecht (2000)

10.1186/1687-1847-2014-27

Cite this article as: Zhang et al.: Mild solutions of fractional evolution equations on an unbounded interval. Advances in Difference Equations 2014, 2014:27 